\title{
Cannabidivarin (CBDV) suppresses pentylenetetrazole (PTZ)- induced increases in epilepsy-related gene expression
}

To date, anticonvulsant effects of the plant cannabinoid, cannabidavarin (CBDV), have been reported in several animal models of seizure. However, these behaviourally observed anticonvulsant effects have not been confirmed at the molecular level. To examine changes to epilepsy-related gene expression following chemical convulsant treatment and their subsequent control by phytocannabinoid administration, we behaviourally evaluated effects of CBDV (400 mg/kg, p.o.) on acute, pentylenetetrazole (PTZ: $95 \mathrm{mg} / \mathrm{kg}$, i.p.)-induced seizures, quantified expression levels of several epilepsy-related genes (Fos, Casp 3, Ccl3, Ccl4, Npy , Arc , Penk , Camk2a, Bdnf and Egr1) by qPCR using hippocampal, neocortical and prefrontal cortical tissue samples before examining correlations between expression changes and seizure severity. PTZ treatment alone produced generalised seizures (median: 5.00 ) and significantly increased expression of Fos, Egr1, Arc , Ccl4 and Bdnf . Consistent with previous findings, CBDV significantly decreased PTZ-induced seizure severity (median: 3.25) and increased latency to the first sign of seizure. Furthermore, there were correlations between reductions of seizure severity and mRNA expression of Fos, Egr1, Arc , Ccl4 and Bdnf in the majority of brain regions in the CBDV+PTZ treated group. When CBDV treated animals were grouped into CBDV responders (criterion: seizure severity $\leq 3.25$ ) and nonresponders (criterion: seizure severity >3.25), PTZ-induced increases of Fos, Egr1, Arc , Ccl4 and Bdnf expression were suppressed in CBDV responders. These results provide the first molecular confirmation of behaviourally observed effects of the non-psychoactive, anticonvulsant cannabinoid, CBDV, upon chemically-induced seizures and serve to underscore its suitability for clinical development. 
1 Naoki Amada ${ }^{1,2,3}$, Yuki Yamasaki ${ }^{1,2,3}$, Claire M. Williams ${ }^{2}$ and Benjamin J. Whalley ${ }^{3}$

$2{ }^{1}$ Qs' Research Institute, Otsuka Pharmaceutical, Co. Ltd., 463-10 Kagasuno, Kawauchi-cho,

3 Tokushima 771-0192, Japan, ${ }^{2}$ School of Psychology and Clinical Language Sciences and ${ }^{3}$ School

4 of Chemistry, Food and Nutritional Sciences and Pharmacy, The University of Reading,

5 Whiteknights, Reading, Berkshire, RG6 6AP, UK.

6 Email addresses:

7 Naoki Amada:na_amada@otsuka-europe.com,n.amada@pgr.reading.ac.uk

8 Yuki Yamasaki: y_yamasaki@otsuka-europe.com

9 Claire M. Williams: claire.williams@reading.ac.uk

10 Benjamin J. Whalley: b.j.whalley@reading.ac.uk

11 Corresponding author:

12 Naoki Amada

13 Phone number: $+44(0) 1183787033$

14 Email addresses: na_amada@otsuka-europe.com,n.amada@pgr.reading.ac.uk

15 Postal address: School of Chemistry, Food and Nutritional Sciences and Pharmacy, The

16 University of Reading, Whiteknights, Reading, Berkshire, RG6 6AP, UK. 


\section{Introduction}

18 Epilepsy affects $\sim 1 \%$ of individuals and is often characterized by recurrent seizures. Many 19 treatments are available but more effective and better-tolerated antiepileptic drugs (AEDs) with new mechanisms of actions are needed due to drug resistance $(\sim 35 \%)$ and poor AED side-effect

21 profiles (Kwan \& Brodie 2007).

Several cannabinoids ( $\Delta^{9}$-tetrahydrocannabinol: $\Delta^{9}$-THC, cannabidiol: CBD, $\Delta^{9}-$ tetrahydrocannabivarin: $\Delta^{9}$-THCV and cannabidivarin: CBDV) are anticonvulsant in a variety of animal models of seizure and epilepsy (Consroe \& Wolkin 1977; Hill et al. 2012a; Hill et al. 2010; Jones et al. 2010). Whilst $\mathrm{CB}_{1}$ cannabinoid receptor $\left(\mathrm{CB}_{1} \mathrm{R}\right)$ agonism is anti-epileptiform and anticonvulsant (Chesher \& Jackson 1974; Deshpande et al. 2007b; Wallace et al. 2003; Wallace et al. 2001), the notable psychoactivity associated with $\mathrm{CB}_{1} \mathrm{R}$ activation hinders the prospective clinical utility of this target. However, many plant cannabinoids do not act at $\mathrm{CB}_{1} \mathrm{R}$ and the most promising non-psychoactive anticonvulsant phytocannabinoid studied thus far is $\mathrm{CBD}$, which exerts effects via, as yet unknown, non- $\mathrm{CB}_{1} \mathrm{R}$ mechanisms in vitro, in vivo and in humans (Consroe et al. 1982; Cunha et al. 1980; Jones et al. 2010; Wallace et al. 2001). Because CBD has low affinity for CB1 and CB2 receptors (Pertwee 2008), CBD may exert its effects through different mechanisms. For instance, it is known that CBD can, at a number of different concentrations in vitro, inhibit adenosine uptake, inhibit FAAH (the enzyme primarily responsible for degradation of the endocannabinoid, anandamide), inhibit anandamide reuptake, act as a TRPA1 receptor agonist, a TRPM8 receptor antagonist, a 5-HT $\mathrm{HA}_{1 \mathrm{~A}}$ receptor agonist, a Ttype calcium channel inhibitor and a regulator of intracellular calcium (Izzo et al. 2009).

Here, we have used molecular methods to further investigate the anticonvulsant potential of CBD's propyl analogue, CBDV (Hill et al. 2012a). Although first isolated in 1969 (Vollner et 
42 direct CB2 receptor effects were not shown (Scutt \& Williamson 2007). De Petrocellis reported

43 differential CBDV effects at transient receptor potential (TRP) channels in vitro, noting potent

44 human TRPA1, TRPV1 and TRPV2 agonism and TRPM8 antagonism (De Petrocellis et al.

45 2011; De Petrocellis et al. 2012). CBDV has also been reported to inhibit diacylglycerol lipase-

$46 \alpha$, the primary synthetic enzyme of the endocannabinoid, 2-arachidonoylglycerol (Bisogno et al.

47 2003), in vitro (De Petrocellis et al. 2011). However, 2-AG inhibits status epilepticus-like

48 activity in rat hippocamal neuronal cultures (Deshpande et al. 2007a) such that diacylglycerol

49 lipase- $\alpha$ inhibition is unlikely to be anticonvulsant. Furthermore, inhibition of DAG lipase by

50 CBDV occurs at high micromolar concentrations $\left(\mathrm{IC}_{50}: 16.6 \mu \mathrm{M}\right)$ in vitro which are unlikely to

51 have relevance in vivo making it unlikely that CBDV exerts anticonvulsant effects via this route.

52 Although the pharmacological relevance of these effects remains unconfirmed in vivo and the

53 targets identified have not yet been linked to epilepsy, they illustrate an emergent role for

54 multiple, non-CB receptor targets of phytocannabinoids (Hill et al. 2012b; Pertwee 2010).

55 Furthermore, unlike $\Delta^{9}$-THC, anticonvulsant doses of CBDV exert no detectable effects upon

56 motor function (Hill et al. 2012a) which further supports the assertion that its effects are not

$57 \quad \mathrm{CB}_{1} \mathrm{R}$-mediated.

58 Despite our earlier report showing significant anticonvulsant effects of CBDV in animal 59 models of acute seizure (Hill et al. 2012a), molecular validation of these effects has not yet been 60 undertaken. Here, we evaluated CBDV's effect (p.o.) on pentylenetetrazole (PTZ)-induced 61 seizures and quantified expression levels of several epilepsy-related genes in tissue from

62 hippocampus, neocortex and prefrontal cortex. Genes of interest were selected on the basis that:

63 i) their expression was significantly changed in previously published gene expression microarray

64 results from people with epilepsy (PWE) (Helbig et al. 2008; Jamali et al. 2006; van Gassen et al.

65 2008) and animal models of epilepsy (Elliott et al. 2003; Gorter et al. 2006; Gorter et al. 2007; 
66 Okamoto et al. 2010) and ii) published results (Johnson et al. 2011; Link et al. 1995; McCarthy et

67 al. 1998; Nanda \& Mack 2000; Saffen et al. 1988; Sola et al. 1998; Zhu \& Inturrisi 1993) 68 suggested that expression changes were acute (within a few hours of seizure), making them 69 suitable for study in a model of acute seizure. On this basis, Early growth response 1 (Egr1), 70 Activity-regulated cytoskeleton-associated protein (Arc), Chemokine (C-C motif) ligand 3 71 (Ccl3), Chemokine (C-C motif) ligand 4 (Ccl4), Brain derived neurotrophic factor (Bdnf), 72 Proenkephalin (Penk) and Neuropeptide Y (Npy) and the downregulated gene, 73 Calcium/calmodulin-dependent protein kinase II alpha (Camk2a) were chosen. FBJ osteosarcoma 74 oncogene (Fos) and Caspase 3 (Casp3) were also selected due to the former's increased 75 expression in brain regions including hippocampus following experimentally induced seizures 76 (e.g. via PTZ) (Popovici et al. 1990; Saffen et al. 1988) and the latter as a result of increased 77 expression in resected neocortex from people with temporal lobe epilepsy (Henshall et al. 2000). 


\section{Material and methods}

\section{2.1. Animals.}

80 Experiments were conducted in accordance with UK Home Office regulations (Animals

81 (Scientific Procedures) Act, 1986). A total of 51 Wistar-Kyoto rats (Harlan, UK; 3-4 weeks old)

82 were used in this study and ARRIVE guidelines complied with. Animals were group housed in 83 cages of five with water and food supplied ad libitum. Temperature and humidity were 84 maintained at $21^{\circ} \mathrm{C}$ and $55 \pm 10 \%$ respectively.

\subsection{Drug administration}

87 Seizures were induced using PTZ (Sigma, Poole, United Kingdom). After overnight fasting, rats 88 received either vehicle $(20 \%$ solutol (Sigma) in $0.9 \% \mathrm{w} / \mathrm{v} \mathrm{NaCl})$ or $\mathrm{CBDV}\left(400 \mathrm{mg} \mathrm{kg}^{-1}\right.$; GW 89 Pharmaceuticals Ltd., Salisbury, UK) in vehicle by oral gavage. Three and a half hours after 90 vehicle or CBDV administration, rats were challenged (i.p.) with saline or PTZ $\left(95 \mathrm{mg} \mathrm{kg}^{-1}\right)$ and

91 behaviour monitored for 1 hour. Animals were euthanised by $\mathrm{CO}_{2}$ overdose and brains 92 immediately removed. Whole hippocampi, neocortices and prefrontal cortices were isolated, 93 snap-frozen in liquid nitrogen and stored at $-80{ }^{\circ} \mathrm{C}$ until RNA extraction.

\section{2.3. Analysis of seizure behaviours}

95 Seizure behaviour was video recorded and responses coded exactly as described previously (Hill 96 et al. 2012a). Responses were coded using the following modified Racine seizure severity scale:

97 0, normal behaviour; 1, isolated myoclonic jerks; 2, atypical clonic seizure; 3, fully developed 98 bilateral forelimb clonus; 3.5 , forelimb clonus with tonic component and body twist; 4 , tonic-

99 clonic seizure with suppressed tonic phase; 5, fully developed tonic-clonic seizure. Latency to 100 the first sign of seizure was also recorded. 
102 Gene expression was quantified in rat hippocampus, prefrontal cortex and neocortex for four

103 experimental groups: vehicle + saline treated $(n=5)$, vehicle + PTZ treated $(n=7)$, CBDV + saline

104 treated $(n=5)$ and CBDV + PTZ treated $(n=7)$. Total RNA was extracted using an miRNeasy Mini

105 kit (Qiagen, West Sussex, UK), following the manufacturer's protocol. RNA purity was assessed 106 spectrometrically at 260/280 nm. RNA integrity was determined by gel electrophoresis. A 107 28S:18S rRNA ratio of $\sim 2: 1$ was taken to indicate intact RNA.

108 Total RNA $(0.5 \mu \mathrm{g})$ was reverse-transcribed into cDNA using High Capacity cDNA Reverse

109 Transcription Kits (Applied Biosystems). qPCR assays were carried out in a volume of $14 \mu 1$, 110 containing $5 \mu \mathrm{l}$ cDNA, $2 \mu \mathrm{l} 2.5 \mu \mathrm{M}$ primer mix (forward and reverse primers) and $7 \mu 1$ 111 QuantiTect SYBR Green QPCR 2× Master Mix (Qiagen, West Sussex, UK). Samples were 112 processed for 40 cycles on a StepOnePlus ${ }^{\mathrm{TM}}$ (Applied Biosystems, Foster City, CA, USA) as 113 follows: denaturation at $95{ }^{\circ} \mathrm{C}$ for 15 minutes (one cycle), 40 cycles of denaturation at $95{ }^{\circ} \mathrm{C}$ for 11415 seconds and annealing at $60{ }^{\circ} \mathrm{C}$ for 1 minute. All samples were analysed in the same plate in a 115 single PCR run and quantification was based on the standard curve method. Standard curves were 116 constructed using cDNA solution diluted fivefold in series for a total of five dilutions and 117 consisted of a mixture of cDNA equally from hippocampus, prefrontal cortex and neocortex of all 118 animals. Sample cDNA concentrations were expressed relative to the concentration of the 119 standard curves. Normalisation of quantitative data was based on a housekeeping gene, $\beta$-actin. 120 Values are expressed as a percentage of control (mean of the vehicle + saline group). The 121 following primers were used (parenthesised values are forward and reverse sequence and 122 amplicon length respectively): Ccl3 (5'-TGCCCTTGCTGTTCTTCTCTGC-3', 5’123 TAGGAGAAGCAGCAGGCAGTCG-3', 96), Ccl4 (5'-CGCCTTCTGCGATTCAGTGC-3', 5'-

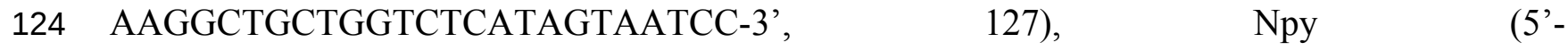
125 TCGTGtGtTtGGGCATtCtGGC-3', 5'-TGtAGTGTCGCAGAGCGGAGTAG-3', 111), Arc 
(5'-AGGCACTCACGCCTGCTCTTAC-3'， 5'-TCAGCCCCAGCTCAATCAAGTCC-3'， 146),

127 Bdnf (5'-AGCCTCCTCTGCTCTTTCTGCTG-3'， 5'-TATCTGCCGCTGTGACCCACTC-3',

$128 \quad$ 150), $\quad$ Egr1 (5'-AGCCTTCGCTCACTCCACTATCC-3',

129 GCGGCTGGgtTtGatGaGTTGG-3', 113), Penk (5'-

130 CCAACTCCTCCGACCTGCTGAAAG-3', 5'-AAGCCCCCATACCTCTTGCTCGTG-3', 121)

131 and Camk2a (5'-TGAGAGCACCAACACCACCATCG-3',

132 TGTCATTCCAGGGTCGCACATCTTC-3', 142), Fos (5'-TGCGTTGCAGACCGAGATTGC-

133 3', 5'-AGCCCAGGTCATTGGGGATCTTG-3', 104), Casp3 (5'-

134 TTGCGCCATGCTGAAACTGTACG-3', 5'-AAAGTGGCGTCCAGGGAGAAGG-3', 111) and

135 B-Actin (5'-CTCTATCCTGGCCTCACTGTCCACC-3', 5'-

136 AAACGCAGCTCAGTAACAGTCCGC-3', 124). Primers were designed using NCBI/Primer-

137 BLAST (http://www.ncbi.nlm.nih.gov/tools/primer-blast/).

138 2.5. Statistics

139 CBDV effects upon seizure severity and onset latency were assessed by comparing vehicle + PTZ

140 treated and CBDV+PTZ treated groups using a two-tailed Mann-Whitney test and a two-tailed t-

141 test, respectively. Subsequently, animals in the CBDV+PTZ treated group were divided

142 according to median seizure severity score into CBDV 'responders' (criterion: seizure severity $\leq$

143 median) and 'non-responders' (criterion: seizure severity $>$ median) to permit a preliminary

144 subgroup analysis of CBDV effects in these two groups without statistical analysis on subgroups.

145 In qPCR analysis, differences of mRNA expressions between treatment groups were analysed in

146 each brain region using one-way analysis of variance (one-way ANOVA) followed by Tukey's

147 test. Correlations between seizure severity and mRNA expression in the CBDV+PTZ treated

148 group were analysed using Spearman's rank correlation coefficient. A preliminary assessment of

149 gene expression changes for CBDV 'responders' and 'non-responders' was performed, in which 
150 differences of mRNA expressions between the vehicle+PTZ treated and the CBDV responder or

151 non-responder subgroups were analysed in each brain region by two-tailed t-test. Since samples

152 from each brain region were analysed on physically separate PCR plates, no comparisons of

153 seizure or drug effects between brain areas were made. Differences were considered statistically

154 significant when the $\mathrm{P} \leq 0.05$. 


\subsection{Anticonvulsant effects of CBDV on PTZ-induced acute seizures}

$157400 \mathrm{mg} \mathrm{kg}^{-1} \mathrm{CBDV}$ significantly decreased seizure severity (vehicle: 5; CBDV: 3.25; $\mathrm{P}<0.05$ )

158 and increased latency to the first seizure sign (vehicle: 60 s; CBDV: 272 s; P $<0.05$; Figure $1 \mathrm{~A} \&$

159 1B). Responses of $\mathrm{CBDV}+\mathrm{PTZ}$ animals sub-grouped into $\mathrm{CBDV}$ responders (criterion: seizure 160 severity $\leq 3.25 ; n=10$ ) and non-responders (criterion: seizure severity $>3.25 ; n=10$ ) showed clear 161 behavioural differences (Figure 1C \& 1D) where CBDV responders exhibit lower seizure 162 severity and increased onset latency.

3.2. Effects of PTZ treatment on $m R N A$ expression of epilepsy-related genes in the hippocampus, neocortex and prefrontal cortex

165 PTZ treatment significantly upregulated Fos mRNA expression in neocortex $(\mathrm{P}=0.0001)$ and 166 prefrontal cortex ( $\mathrm{P}=0.0003$; Table 1) whilst hippocampal Fos mRNA expression only showed a trend to increase $(\mathrm{P}=0.1089)$. Egr1 mRNA expression was significantly upregulated by $\mathrm{PTZ}$ treatment in the hippocampus $(\mathrm{P}=0.0244)$, neocortex $(\mathrm{P}=0.0001)$ and prefrontal cortex $(\mathrm{P}<0.0001)$ whilst Arc mRNA expression was also significantly upregulated by PTZ treatment in the hippocampus $(\mathrm{P}=0.0374)$, neocortex $(\mathrm{P}=0.0039)$ and prefrontal cortex $(\mathrm{P}=0.0038)$. Expression of Cc14 mRNA was significantly upregulated only in the prefrontal cortex $(\mathrm{P}=0.0220)$ by PTZ

172 treatment. Trends toward an increase of Ccl4 mRNA expression in the hippocampus $(\mathrm{P}=0.1720)$ 173 and neocortex $(\mathrm{P}=0.1093)$ by $\mathrm{PTZ}$ treatment were seen. Expression of Bdnf mRNA was 174 significantly upregulated in the neocortex $(\mathrm{P}=0.0308)$ and prefrontal cortex $(\mathrm{P}=0.0345)$ but only a 175 trend towards increased expression in the hippocampus was seen $(\mathrm{P}=0.0564)$. mRNA expression 176 of Casp3, Npy, Penk, Ccl3 and Camk2a were not significantly changed by any treatment. 
178

179

180

181

182

183

184

185

186

187

188

189

190

191

192

193

194

195

196

197

198

199

200

201

3.3. Effects of CBDV upon PTZ-induced $m R N A$ expression of epilepsy-related genes in the hippocampus, neocortex and prefrontal cortex

Fos and Egr1 mRNA expression were significantly upregulated in the neocortex $(\mathrm{P}=0.0201$ and $\mathrm{P}=0.0033$, respectively) and the prefrontal cortex $(\mathrm{P}=0.0156$ and $\mathrm{P}=0.0023$, respectively $)$ in the $\mathrm{CBDV}+\mathrm{PTZ}$ treated group. Although there were no statistically significant changes in the expression levels of any other genes between the vehicle+saline and CBDV + PTZ treated groups which suggests an inhibitory effect of CBDV on PTZ-induced upregulation of gene expression, neither were statistically significant differences in gene expression levels between the vehicle $+\mathrm{PTZ}$ and $\mathrm{CBDV}+\mathrm{PTZ}$ treated groups found. However, when potential correlations between the behavioural measure of seizure severity and mRNA expression levels of Fos, Egr1, Arc, Bdnf and Ccl4 in the CBDV+PTZ treated group were examined using Spearman's rank correlation coefficient, mRNA expression levels of these genes were highly correlated with seizure severity in the majority of brain regions examined (Figure 2: hippocampus, Figure 3: neocortex and Figure 4: prefrontal cortex). Fos mRNA expression correlated with seizure severity in the hippocampus $\left(\mathrm{R}^{2}=0.91, \mathrm{P}=0.0008\right)$, neocortex $\left(\mathrm{R}^{2}=0.91, \mathrm{P}=0.0008\right)$ and prefrontal cortex $\left(\mathrm{R}^{2}=0.91, \mathrm{P}=0.0008\right)$ of the $\mathrm{CBDV}+\mathrm{PTZ}$ treated group. Egr1 mRNA expression was correlated with seizure severity only in the hippocampus $\left(\mathrm{R}^{2}=0.91, \mathrm{P}=0.0008\right)$ whilst Arc mRNA expression was correlated with seizure severity in the hippocampus $\left(\mathrm{R}^{2}=0.91, \mathrm{P}=0.0008\right)$, neocortex $\left(\mathrm{R}^{2}=0.91, \mathrm{P}=0.0008\right)$ and prefrontal cortex $\left(\mathrm{R}^{2}=0.71, \mathrm{P}=0.0175\right)$. Bdnf mRNA expression was correlated with seizure severity in the hippocampus $\left(\mathrm{R}^{2}=0.71, \mathrm{P}=0.0175\right)$ and neocortex $\left(\mathrm{R}^{2}=0.65\right.$, $\mathrm{P}=0.0291$ ) whilst Ccl4 mRNA expression was correlated with seizure severity in the hippocampus $\left(\mathrm{R}^{2}=0.91, \mathrm{P}=0.0008\right)$, neocortex $\left(\mathrm{R}^{2}=0.71, \mathrm{P}=0.0175\right)$ and prefrontal cortex $\left(\mathrm{R}^{2}=0.71, \mathrm{P}=0.0175\right)$. Together, these suggest a possible contribution of the anti-convulsant effects of CBDV in reduction of mRNA expression of Fos, Egr1, Arc, Bdnf and Cc14. 
202

203

204 Consistent with differing behavioural patterns observed between CBDV responder and non-

205

206

207

208

209

210

211

212

213

214

215

216

217

218

219

220

221

222

223

224

225

3.4. Effects of CBDV treatment on the PTZ-induced increases of the epilepsy-related genes in $C B D V$ responders

responder subgroups, alterations in gene expression were also seen. Importantly, changes in gene expression levels between the vehicle+PTZ and the CBDV responder subgroups were most obvious, with few changes seen in gene expression levels between vehicle+PTZ and the CBDV+PTZ non-responder subgroups. Importantly, PTZ-induced increases in gene expression were most reliably suppressed in the hippocampus of CBDV responders, with less obvious suppression in prefrontal cortex and neocortex. The PTZ-induced increase of Fos mRNA expression in CBDV responders was suppressed in the neocortex $(\mathrm{P}=0.0274)$ and the prefrontal cortex $(\mathrm{P}=0.0337)$, and there was a strong trend towards a decrease in the hippocampus $(\mathrm{P}=0.0579 ;$ Figure 5A). The PTZ-induced increase of Egr1 mRNA expression was suppressed in the hippocampus $(\mathrm{P}=0.0234)$ of $\mathrm{CBDV}$ responders, but less obviously so in the neocortex $(\mathrm{P}=0.1837)$ and the prefrontal cortex $(\mathrm{P}=0.1038$; Figure 5B$)$. The increase in Arc mRNA expression induced by PTZ treatment was also suppressed in the hippocampus $(\mathrm{P}=0.0221)$ of CBDV responders, and there were strong trends towards decreases in the neocortex $(\mathrm{P}=0.0643)$ and the prefrontal cortex $(\mathrm{P}=0.0879$; Figure $5 \mathrm{C})$. The increase of Bdnf mRNA expression following PTZ treatment was most suppressed in the hippocampus $(\mathrm{P}=0.0441)$ of $\mathrm{CBDV}$ responders whilst less decreases were seen in the neocortex $(\mathrm{P}=0.1099)$ and prefrontal cortex $(\mathrm{P}=0.4128$; Figure 5D). Finally the $\mathrm{PTZ}$-induced increase of $\mathrm{Ccl} 4 \mathrm{mRNA}$ expression was suppressed in the hippocampus $(\mathrm{P}=0.0323)$ and the prefrontal cortex $(\mathrm{P}=0.0459)$, and there was a strong trend towards a decrease in the neocortex $(\mathrm{P}=0.0942$; Figure 5E). On the other hand, neither statistically significant decreases nor trends towards decreases in the gene expressions were found in the CBDV non-responder subgroup. 


\section{Discussion}

227 PTZ treatment upregulated (significant increase or statistically strong trend to increase) mRNA expression coding for Fos, Egr1, Arc, Ccl4 and Bdnf in all brain regions tested. Clear correlations

229 between seizure severity and mRNA expression were observed for these genes in the majority of brain regions of $\mathrm{CBDV}+\mathrm{PTZ}$ treated animals and mRNA expression of these genes was suppressed in the majority of brain regions examined from the CBDV responder subgroup. Upregulation of Fos and Egr1 mRNA expression following PTZ treatment has previously been reported in rat hippocampus (Saffen et al. 1988) and both Fos and Egr1 are transcription factors belonging to IEG (immediate early gene) family which is transiently and rapidly activated following a variety of cellular stimuli. IEGs can identify activated neurons and brain circuits since seizure activity, and other excitatory stimuli, can induce rapid and transient Fos expression increases (Herrera \& Robertson 1996), making it a useful metabolic marker for brain activity (Dragunow \& Faull 1989). Fos expression level in the brain is typically low under basal conditions and is induced in response to extracellular signals such as ions, neurotransmitters, growth factors and drugs and is closely linked to the induction of transcription of other genes (Kovacs 2008). Fos induction also correlates with the mossy fibre sprouting (Kiessling \& Gass 1993; Popovici et al. 1990) that occurs during epileptogenesis and may play a role in the subsequent manifestation of seizure symptoms. Like Fos, Egr-1 also activates transcription of

244 other genes (Beckmann et al. 1997; Christy \& Nathans 1989) and is considered to play an 245 important role in neuronal plasticity (Knapska \& Kaczmarek 2004). Furthermore, the expression 246 of Fos and Egr1 in seizure onset regions in PWE strongly correlates with interictal spiking [10]. 247 Thus, suppression of Fos and Egr1 mRNA expression are consistent with ameliorative drug 248 effects on seizures, epileptogenesis and/or epilepsy. In addition, increased Arc mRNA expression 249 in rat hippocampus (0.5-4 hours) and cortex (0.5-1 hour) after PTZ treatment has also been 250 reported (Link et al. 1995). It has been reported that newly synthesised Arc mRNA is selectively 
251 localised in active dendritic segments and that Arc plays a role in activity-dependent plasticity of

252 dendrites (Lyford et al. 1995; Steward et al. 1998). Arc is induced by hippocampal seizures, and

253 glutamatergic neurons increase Arc expression in response to increased synaptic activity (Korb \&

254 Finkbeiner 2011), implying a relationship between seizure activity and Arc expression. Ccl4 is

255 a proinflammatory chemokine that is known as a chemo-attractant for monocytes and $\mathrm{T}$ cells and

256 has been suggested to play a part in various nervous system pathologies such as inflammation,

257 trauma, ischemia and multiple sclerosis (Semple et al. 2010). Although a relationship between

258 CCL4 and epilepsy is unclear, a relationship between epilepsy and immune response has been

259 suggested (Vezzani \& Granata 2005). Moreover, increased Cc14 mRNA expression has been

260 reported in rat hippocampus and temporal lobe tissue following status epilepticus events

261 triggered by electrical stimulation of the amygdala (Guzik-Kornacka et al. 2011). In the present

262 study, PTZ-induced increase of Ccl4 expression was suppressed in CBDV responders, although

263 whether this is a direct anti-inflammatory effect of CBDV or an indirect effect of reduced seizure

264 severity remains unknown. Increased expression of mRNA coding for Bdnf was confirmed in rat

265 hippocampus after PTZ treatment (Nanda \& Mack 2000). BDNF is one of many neurotrophic

266 factors and is known to promote survival and growth of a variety of neurons in addition to

267 strengthening excitatory (glutamatergic) synapses (Binder \& Scharfman 2004). BDNF is

268 involved in the control of hippocampal plasticity and is thought to play an important role in

269 epileptogenesis and in temporal lobe epilepsy (Binder et al. 2001; Scharfman 2002), suggesting

270 therapeutic importance for control of Bdnf expression.

\section{5. Conclusions}

272 We have confirmed upregulation of mRNA expression coding for Fos, Egr1, Arc, Ccl4 and Bdnf

273 in the brains of rats treated with PTZ and shown that PTZ-induced increases of mRNA

274 expression for these genes were suppressed in CBDV responders, and not animals that failed to 
275 respond to $\mathrm{CBDV}$ treatment. Overall, we provide molecular evidence that directly supports 276 behavioural evidence that CBDV exerts significant anticonvulsant effects via oral and other 277 routes of administration (Hill et al. 2012a). Whether gene expression changes demonstrated here 278 also underlie cellular and molecular mechanisms by which CBDV exerts its anticonvulsant effect 279 presently remains unknown. However, these results provide important acute biomarkers for 280 additional investigation in models of the progressive disorder and following longer term CBDV 281 treatment.

\section{Acknowledgement}

283 We thank both companies for research sponsorship and thank GW Pharmaceuticals Plc. for the 284 provision of CBDV. 
References

286 Beckmann AM, Davidson MS, Goodenough S, and Wilce PA. 1997. Differential expression of 287 good thing? Trends in Neurosciences 24:47-53.

291

Binder DK, and Scharfman HE. 2004. Brain-derived neurotrophic factor. Growth Factors 292 $22: 123-131$.

Bisogno T, Howell F, Williams G, Minassi A, Cascio MG, Ligresti A, Matias I, SchianoMoriello A, Paul P, Williams EJ et al. . 2003. Cloning of the first sn1-DAG lipases points to the spatial and temporal regulation of endocannabinoid signaling in the brain. $J$ Cell Biol 163:463-468.

Chesher GB, and Jackson DM. 1974. Anticonvulsant effects of cannabinoids in mice: drug interactions within cannabinoids and cannabinoid interactions with phenytoin. Psychopharmacologia 37:255-264.

300

301

Christy B, and Nathans D. 1989. DNA binding site of the growth factor-inducible protein Zif268. Proc Natl Acad Sci US A 86:8737-8741.

302

Consroe P, Benedito MA, Leite JR, Carlini EA, and Mechoulam R. 1982. Effects of cannabidiol 303 304 on behavioral seizures caused by convulsant drugs or current in mice. Eur J Pharmacol

Consroe P, and Wolkin A. 1977. Cannabidiol--antiepileptic drug comparisons and interactions in 306 experimentally induced seizures in rats. J Pharmacol Exp Ther 201:26-32.

307 Cunha JM, Carlini EA, Pereira AE, Ramos OL, Pimentel C, Gagliardi R, Sanvito WL, Lander N, 308 and Mechoulam R. 1980. Chronic administration of cannabidiol to healthy volunteers and 309 epileptic patients. Pharmacology 21:175-185. 
310

311

312

313

314

315

316

317

318

319

320

321

322

323

324

325

326

327

328

329

330

331

332

333

De Petrocellis L, Ligresti A, Moriello AS, Allara M, Bisogno T, Petrosino S, Stott CG, and Di Marzo V. 2011. Effects of cannabinoids and cannabinoid-enriched Cannabis extracts on TRP channels and endocannabinoid metabolic enzymes. Br J Pharmacol 163:1479-1494.

De Petrocellis L, Orlando P, Moriello AS, Aviello G, Stott C, Izzo AA, and Di Marzo V. 2012. Cannabinoid actions at TRPV channels: effects on TRPV3 and TRPV4 and their potential relevance to gastrointestinal inflammation. Acta Physiol (Oxf) 204:255-266.

Deshpande LS, Blair RE, Ziobro JM, Sombati S, Martin BR, and DeLorenzo RJ. 2007a. Endocannabinoids block status epilepticus in cultured hippocampal neurons. Eur $J$ Pharmacol 558:52-59.

Deshpande LS, Sombati S, Blair RE, Carter DS, Martin BR, and DeLorenzo RJ. $2007 \mathrm{~b}$. Cannabinoid CB1 receptor antagonists cause status epilepticus-like activity in the hippocampal neuronal culture model of acquired epilepsy. Neurosci Lett 411:11-16.

Dragunow M, and Faull R. 1989. The use of c-fos as a metabolic marker in neuronal pathway tracing. J Neurosci Methods 29:261-265.

Elliott RC, Miles MF, and Lowenstein DH. 2003. Overlapping microarray profiles of dentate gyrus gene expression during development- and epilepsy-associated neurogenesis and axon outgrowth. J Neurosci 23:2218-2227.

Gorter JA, van Vliet EA, Aronica E, Breit T, Rauwerda H, Lopes da Silva FH, and Wadman WJ. 2006. Potential new antiepileptogenic targets indicated by microarray analysis in a rat model for temporal lobe epilepsy. J Neurosci 26:11083-11110.

Gorter JA, Van Vliet EA, Rauwerda H, Breit T, Stad R, van Schaik L, Vreugdenhil E, Redeker S, Hendriksen E, Aronica E et al. . 2007. Dynamic changes of proteases and protease inhibitors revealed by microarray analysis in CA3 and entorhinal cortex during epileptogenesis in the rat. Epilepsia 48 Suppl 5:53-64. 
334 Guzik-Kornacka A, Sliwa A, Plucinska G, and Lukasiuk K. 2011. Status epilepticus evokes 335 prolonged increase in the expression of CCL3 and CCL4 mRNA and protein in the rat $336 \quad$ brain. Acta Neurobiol Exp (Wars) 71:193-207.

337 Helbig I, Matigian NA, Vadlamudi L, Lawrence KM, Bayly MA, Bain SM, Diyagama D, 338 Scheffer IE, Mulley JC, Holloway AJ et al. . 2008. Gene expression analysis in absence $339 \quad$ epilepsy using a monozygotic twin design. Epilepsia 49:1546-1554.

340 Henshall DC, Clark RS, Adelson PD, Chen M, Watkins SC, and Simon RP. 2000. Alterations in 341 bcl-2 and caspase gene family protein expression in human temporal lobe epilepsy. $342 \quad$ Neurology 55:250-257.

343 Herrera DG, and Robertson HA. 1996. Activation of c-fos in the brain. Prog Neurobiol 50:83$344 \quad 107$.

345 Hill AJ, Mercier MS, Hill TD, Glyn SE, Jones NA, Yamasaki Y, Futamura T, Duncan M, Stott 346 CG, Stephens GJ et al. . 2012a. Cannabidivarin is anticonvulsant in mouse and rat. $\mathrm{Br} J$ $347 \quad$ Pharmacol 167:1629-1642.

348 Hill AJ, Weston SE, Jones NA, Smith I, Bevan SA, Williamson EM, Stephens GJ, Williams CM, and Whalley BJ. 2010. Delta-Tetrahydrocannabivarin suppresses in vitro epileptiform and in vivo seizure activity in adult rats. Epilepsia 51:1522-1532.

351

Hill AJ, Williams CM, Whalley BJ, and Stephens GJ. 2012b. Phytocannabinoids as novel 352 therapeutic agents in CNS disorders. Pharmacol Ther 133:79-97.

353 Izzo AA, Borrelli F, Capasso R, Di Marzo V, and Mechoulam R. 2009. Non-psychotropic plant 354 cannabinoids: new therapeutic opportunities from an ancient herb. Trends Pharmacol Sci 355 30:515-527.

356 Jamali S, Bartolomei F, Robaglia-Schlupp A, Massacrier A, Peragut JC, Regis J, Dufour H, 357 Ravid R, Roll P, Pereira S et al. . 2006. Large-scale expression study of human mesial 
temporal lobe epilepsy: evidence for dysregulation of the neurotransmission and complement systems in the entorhinal cortex. Brain 129:625-641.

360

361

362

363

364

365

366

367

368

369

370

371

372

373

374

375

376

377

378

379

380

381

Johnson EA, Dao TL, Guignet MA, Geddes CE, Koemeter-Cox AI, and Kan RK. 2011. Increased expression of the chemokines CXCL1 and MIP-1alpha by resident brain cells precedes neutrophil infiltration in the brain following prolonged soman-induced status epilepticus in rats. $J$ Neuroinflammation 8:41.

Jones NA, Hill AJ, Smith I, Bevan SA, Williams CM, Whalley BJ, and Stephens GJ. 2010. Cannabidiol displays antiepileptiform and antiseizure properties in vitro and in vivo. $J$ Pharmacol Exp Ther 332:569-577.

Kiessling M, and Gass P. 1993. Immediate early gene expression in experimental epilepsy. Brain Pathol 3:381-393.

Knapska E, and Kaczmarek L. 2004. A gene for neuronal plasticity in the mammalian brain: Zif268/Egr-1/NGFI-A/Krox-24/TIS8/ZENK? Prog Neurobiol 74:183-211.

Korb E, and Finkbeiner S. 2011. Arc in synaptic plasticity: from gene to behavior. Trends in Neurosciences 34:591-598.

Kovacs KJ. 2008. Measurement of immediate-early gene activation- c-fos and beyond. $J$ Neuroendocrinol 20:665-672.

Kwan P, and Brodie MJ. 2007. Emerging drugs for epilepsy. Expert Opin Emerg Drugs 12:407422.

Link W, Konietzko U, Kauselmann G, Krug M, Schwanke B, Frey U, and Kuhl D. 1995. Somatodendritic expression of an immediate early gene is regulated by synaptic activity. Proc Natl Acad Sci U S A 92:5734-5738.

Lyford GL, Yamagata K, Kaufmann WE, Barnes CA, Sanders LK, Copeland NG, Gilbert DJ, Jenkins NA, Lanahan AA, and Worley PF. 1995. Arc, a growth factor and activity- 
regulated gene, encodes a novel cytoskeleton-associated protein that is enriched in neuronal dendrites. Neuron 14:433-445.

384 McCarthy JB, Walker M, Pierce J, Camp P, and White JD. 1998. Biosynthesis and metabolism of native and oxidized neuropeptide $\mathrm{Y}$ in the hippocampal mossy fiber system. $J$ Neurochem 70:1950-1963.

Nanda SA, and Mack KJ. 2000. Seizures and sensory stimulation result in different patterns of brain derived neurotrophic factor protein expression in the barrel cortex and hippocampus. Brain Res Mol Brain Res 78:1-14.

Okamoto OK, Janjoppi L, Bonone FM, Pansani AP, da Silva AV, Scorza FA, and Cavalheiro EA. 2010. Whole transcriptome analysis of the hippocampus: toward a molecular portrait of epileptogenesis. BMC Genomics 11:230.

Pertwee RG. 2008. The diverse CB1 and CB2 receptor pharmacology of three plant cannabinoids: delta9-tetrahydrocannabinol, cannabidiol and delta9tetrahydrocannabivarin. Br J Pharmacol 153:199-215.

Pertwee RG. 2010. Receptors and channels targeted by synthetic cannabinoid receptor agonists and antagonists. Curr Med Chem 17:1360-1381.

398 Popovici T, Represa A, Crepel V, Barbin G, Beaudoin M, and Ben-Ari Y. 1990. Effects of kainic 399 acid-induced seizures and ischemia on c-fos-like proteins in rat brain. Brain Res 536:183194.

401 Saffen DW, Cole AJ, Worley PF, Christy BA, Ryder K, and Baraban JM. 1988. Convulsant402 403 induced increase in transcription factor messenger RNAs in rat brain. Proc Natl Acad Sci U S A 85:7795-7799.

404 Scharfman H. 2002. Does BDNF Contribute to Temporal Lobe Epilepsy? Epilepsy Curr 2:92-94. 405 Scutt A, and Williamson EM. 2007. Cannabinoids stimulate fibroblastic colony formation by 406 bone marrow cells indirectly via CB2 receptors. Calcif Tissue Int 80:50-59. 
407 Semple BD, Kossmann T, and Morganti-Kossmann MC. 2010. Role of chemokines in CNS 408 health and pathology: a focus on the CCL2/CCR2 and CXCL8/CXCR2 networks. $J$ $409 \quad$ Cereb Blood Flow Metab 30:459-473.

410 Sola C, Tusell JM, and Serratosa J. 1998. Decreased expression of calmodulin kinase II and 411 calcineurin messenger RNAs in the mouse hippocampus after kainic acid-induced 412 seizures. J Neurochem 70:1600-1608.

413 Steward O, Wallace CS, Lyford GL, and Worley PF. 1998. Synaptic activation causes the mRNA 414 for the IEG Arc to localize selectively near activated postsynaptic sites on dendrites. 415 Neuron 21:741-751.

van Gassen KL, de Wit M, Koerkamp MJ, Rensen MG, van Rijen PC, Holstege FC, Lindhout D, and de Graan PN. 2008. Possible role of the innate immunity in temporal lobe epilepsy. Epilepsia 49:1055-1065.

Vezzani A, and Granata T. 2005. Brain inflammation in epilepsy: experimental and clinical 420 evidence. Epilepsia 46:1724-1743.

421 Vollner L, Bieniek D, and Korte F. 1969. [Hashish. XX. Cannabidivarin, a new hashish constituent]. Tetrahedron Lett:145-147.

423 Wallace MJ, Blair RE, Falenski KW, Martin BR, and DeLorenzo RJ. 2003. The endogenous 424 cannabinoid system regulates seizure frequency and duration in a model of temporal lobe epilepsy. J Pharmacol Exp Ther 307:129-137.

426 Wallace MJ, Wiley JL, Martin BR, and DeLorenzo RJ. 2001. Assessment of the role of CB1 receptors in cannabinoid anticonvulsant effects. Eur J Pharmacol 428:51-57.

Zhu YS, and Inturrisi CE. 1993. Metrazole induction of c-fos and proenkephalin gene expression 429 in the rat adrenal and hippocampus: pharmacological characterization. Brain Res Mol 430 Brain Res 20:118-124. 


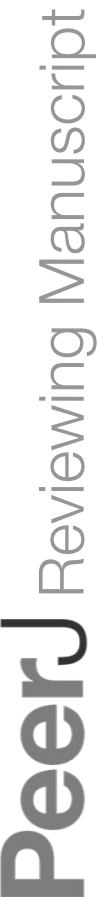

PeerJ reviewing PDF | (v2013:06:586:2:0:NEW 29 Oct 2013) 


\section{Table 1 (on next page)}

Relative mRNA expression levels of epilepsy-related genes in the hippocampus (HIP), neocortex (Nctx) and prefrontal cortex (PFC).

Expression of Fos, Egr1, Arc, Ccl4 and Bdnf were upregulated by PTZ treatment. mRNA levels are presented as a fold change vs mean level of vehicle+saline treated group (data are expressed as mean \pm s.e.m.). Differences between individual groups were assessed by 1 way ANOVA (followed by a Tukey's post-hoc test if warranted). ${ }^{* *}: \mathrm{P}<0.01,{ }^{*}: \mathrm{P}<0.05$ vs vehicle+saline group. 


\begin{tabular}{|c|c|c|c|c|c|c|c|c|}
\hline Gene Official Name & $\begin{array}{c}\text { Gene } \\
\text { Symbol }\end{array}$ & \multirow{4}{*}{$\begin{array}{l}\text { Cellular response to calcium ion, cellular response to } \\
\text { extracellular stimulus, inflammatory response, nervous system } \\
\text { development }\end{array}$} & \multirow{4}{*}{$\begin{array}{c}\begin{array}{c}\text { Brain } \\
\text { Region }\end{array} \\
\text { HIP } \\
\text { Nctx } \\
\text { PFC }\end{array}$} & \multirow{4}{*}{$\begin{array}{c}\begin{array}{c}\text { Vehicle }+ \text { Saline } \\
\text { Fold change } \\
(\mathrm{N}=5)\end{array} \\
1.0 \pm 0.2 \\
1.0 \pm 0.3 \\
1.0 \pm 0.1\end{array}$} & $\begin{array}{l}\text { Vehicle }+ \text { PTZ } \\
\text { Fold change } \\
\quad(\mathrm{N}=7) \\
\end{array}$ & $\begin{array}{l}\text { CBDV + Saline } \\
\text { Fold change } \\
(\mathrm{N}=5)\end{array}$ & \multicolumn{2}{|c|}{$\begin{array}{l}\mathrm{CBDV}+\mathrm{PTZ} \\
\text { Fold change } \\
(\mathrm{N}=7) \\
\end{array}$} \\
\hline \multirow{3}{*}{ FBJ osteosarcoma oncogene } & \multirow{3}{*}{ Fos } & & & & $55.6 \pm 22.2$ & $0.8 \pm 0.1$ & $25.4 \pm 15.0$ & \\
\hline & & & & & $21.5 \pm 3.5 * *$ & $0.7 \pm 0.1$ & $13.2 \pm 2.8 *$ & $*$ \\
\hline & & & & & $20.0 \pm 3.8 * *$ & $0.8 \pm 0.1$ & $13.5 \pm 2.3 *$ & $*$ \\
\hline \multirow{3}{*}{ Caspase 3} & \multirow{3}{*}{ Casp3 } & \multirow{3}{*}{ Apoptosis, intracellular signal transduction } & HIP & $1.0 \pm 0.1$ & $0.9 \pm 0.1$ & $0.9 \pm 0.1$ & $0.9 \pm 0.1$ & \\
\hline & & & Nctx & $1.0 \pm 0.1$ & $1.1 \pm 0.1$ & $1.0 \pm 0.1$ & $1.1 \pm 0.1$ & \\
\hline & & & PFC & $1.0 \pm 0.0$ & $1.1 \pm 0.1$ & $0.9 \pm 0.1$ & $0.9 \pm 0.1$ & \\
\hline \multirow{3}{*}{ Early growth response 1} & \multirow{3}{*}{ Egr1 } & \multirow{3}{*}{$\begin{array}{l}\text { Cellular response to drug, cellular response to growth factor } \\
\text { stimulus, cellular response to steroid hormone stimulus, } \\
\text { circadian rhythm, interleukin-1-mediated signaling pathway }\end{array}$} & HIP & $1.0 \pm 0.0$ & $6.1 \pm 1.5 *$ & $0.8 \pm 0.1$ & $3.6 \pm 1.1$ & \\
\hline & & & Nctx & $1.0 \pm 0.1$ & $3.0 \pm 0.4 * *$ & $0.7 \pm 0.1$ & $2.5 \pm 0.2$ & $* *$ \\
\hline & & & PFC & $1.0 \pm 0.1$ & $2.7 \pm 0.3 * *$ & $0.8 \pm 0.1$ & $2.2 \pm 0.2$ & $* *$ \\
\hline \multirow{3}{*}{$\begin{array}{c}\text { Activity-regulated } \\
\text { cytoskeleton-associated protein }\end{array}$} & \multirow{3}{*}{ Arc } & \multirow{3}{*}{ Regulation of neuronal synaptic plasticity, endocytosis } & HIP & $1.0 \pm 0.1$ & $8.6 \pm 2.5 *$ & $0.8 \pm 0.1$ & $4.2 \pm 1.7$ & \\
\hline & & & Nctx & $1.0 \pm 0.2$ & $5.0 \pm 1.1 * *$ & $0.6 \pm 0.1$ & $3.4 \pm 0.5$ & \\
\hline & & & PFC & $1.0 \pm 0.1$ & $4.4 \pm 0.9 * *$ & $0.7 \pm 0.1$ & $3.0 \pm 0.4$ & \\
\hline \multirow{3}{*}{ Neuropeptide Y } & \multirow{3}{*}{ Npy } & \multirow{3}{*}{$\begin{array}{l}\text { Feeding behavior, negative regulation of blood pressure, } \\
\text { synaptic transmission }\end{array}$} & HIP & $1.0 \pm 0.1$ & $0.9 \pm 0.1$ & $1.0 \pm 0.1$ & $1.0 \pm 0.1$ & \\
\hline & & & Nctx & $1.0 \pm 0.1$ & $1.0 \pm 0.1$ & $1.0 \pm 0.1$ & $1.1 \pm 0.1$ & \\
\hline & & & PFC & $1.0 \pm 0.1$ & $0.9 \pm 0.0$ & $1.0 \pm 0.1$ & $0.9 \pm 0.0$ & \\
\hline \multirow{3}{*}{ Chemokine (C-C motif) ligand 4} & \multirow{3}{*}{ Ccl4 } & \multirow{3}{*}{ Chemotaxis, inflammatory response } & HIP & $1.0 \pm 0.1$ & $16.7 \pm 5.9$ & $0.7 \pm 0.2$ & $7.9 \pm 6.3$ & \\
\hline & & & Nctx & $1.0 \pm 0.3$ & $36.0 \pm 14.8$ & $1.4 \pm 0.3$ & $15.4 \pm 8.8$ & \\
\hline & & & PFC & $1.0 \pm 0.2$ & $13.3 \pm 3.4 *$ & $1.0 \pm 0.2$ & $7.9 \pm 3.0$ & \\
\hline \multirow{3}{*}{ Chemokine (C-C motif) ligand 3} & \multirow{3}{*}{ Ccl3 } & \multirow{3}{*}{$\begin{array}{l}\text { Chemotaxis, elevation of cytosolic calcium ion concentration, } \\
\text { inflammatory response }\end{array}$} & HIP & $1.0 \pm 0.2$ & $8.8 \pm 3.7$ & $1.1 \pm 0.2$ & $5.3 \pm 3.7$ & \\
\hline & & & Nctx & $1.0 \pm 0.2$ & $21.1 \pm 10.5$ & $1.6 \pm 0.2$ & $13.0 \pm 6.2$ & \\
\hline & & & PFC & $1.0 \pm 0.1$ & $16.4 \pm 6.3$ & $1.5 \pm 0.1$ & $13.5 \pm 5.9$ & \\
\hline \multirow{3}{*}{ Brain derived neurotrophic factor } & \multirow{3}{*}{ Bdnf } & \multirow{3}{*}{$\begin{array}{l}\text { Neuron differentiation, positive regulation of long-term } \\
\text { neuronal synaptic plasticity, glutamate secretion }\end{array}$} & HIP & $1.0 \pm 0.1$ & $2.6 \pm 0.6$ & $0.9 \pm 0.1$ & $1.7 \pm 0.3$ & \\
\hline & & & Nctx & $1.0 \pm 0.0$ & $2.5 \pm 0.4 *$ & $0.9 \pm 0.1$ & $2.1 \pm 0.4$ & \\
\hline & & & PFC & $1.0 \pm 0.1$ & $2.1 \pm 0.4 *$ & $1.1 \pm 0.2$ & $1.9 \pm 0.2$ & \\
\hline \multirow{3}{*}{ Proenkephalin } & & & HIP & $1.0 \pm 0.1$ & $1.2 \pm 0.2$ & $1.1 \pm 0.1$ & $1.1 \pm 0.1$ & \\
\hline & & & Nctx & $1.0 \pm 0.2$ & $1.1 \pm 0.2$ & $0.8 \pm 0.1$ & $1.1 \pm 0.1$ & \\
\hline & Penk & Behavioral fear response, sensory perception of pain & PFC & $1.0 \pm 0.2$ & $0.9 \pm 0.2$ & $1.1 \pm 0.2$ & $0.9 \pm 0.2$ & \\
\hline Calcium/calmodulin-dependent protein & Camk2a & Calcium ion transport, ionotropic glutamate receptor signaling & HIP & $1.0 \pm 0.1$ & $0.9 \pm 0.0$ & $0.9 \pm 0.1$ & $0.9 \pm 0.1$ & \\
\hline kinase II alpha & & pathway, protein phosphorylation, regulation of neuronal & Nctx & $1.0 \pm 0.1$ & $0.9 \pm 0.1$ & $1.0 \pm 0.1$ & $1.0 \pm 0.1$ & \\
\hline
\end{tabular}




\section{Figure 1}

Anticonvulsant effects of CBDV on PTZ-induced acute seizures.

A: Plot showing median seizure severity in the vehicle- and CBDV-treated groups following PTZ administration. B: Plot showing latency (seconds) to the first seizure sign in the vehicle- and CBDVtreated groups. C: Seizure severity after sub-grouping CBDV treated group animals into CBDV non-responders and CBDV responders. D: Latency (seconds) to the first seizure sign after subgrouping CBDV treated group animals into CBDV non-responders and CBDV responders. In seizure severity plots, median seizure severity is represented by a thick horizontal line, the 25th and the 75th percentiles are represented by the box and maxima and minima are represented by 'whiskers'. Latency to the first seizure sign was presented as mean \pm SEM. *: $\mathrm{P}<0.05$ by MannWhitney Test vs vehicle group. \#: $\mathrm{P}<0.05$ by t-test vs vehicle group.

A

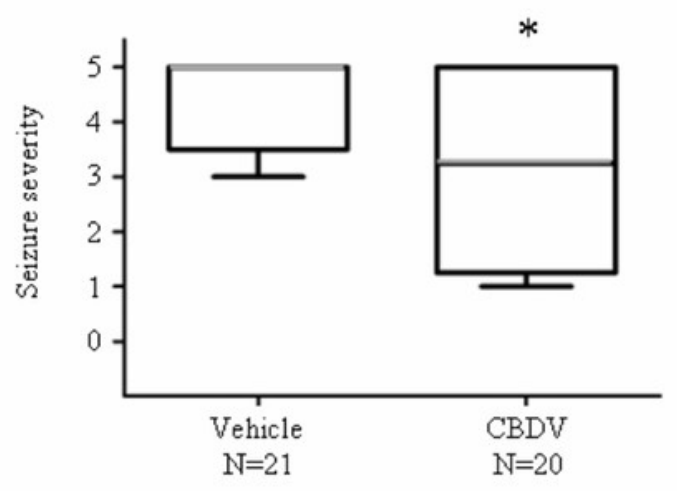

$\mathrm{C}$

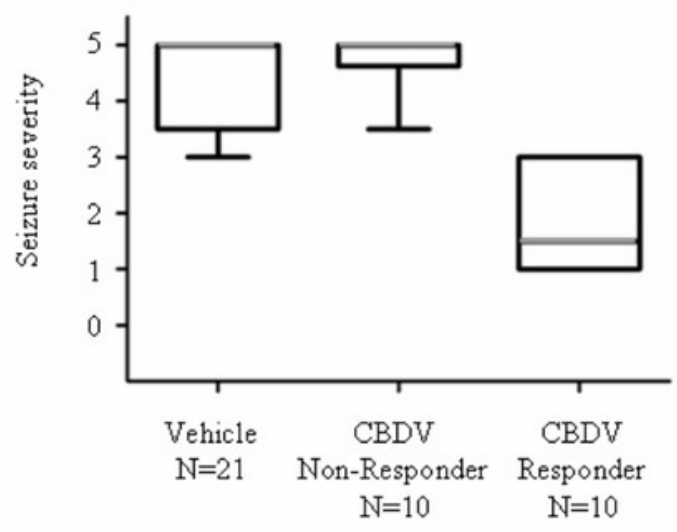

$\mathrm{B}$

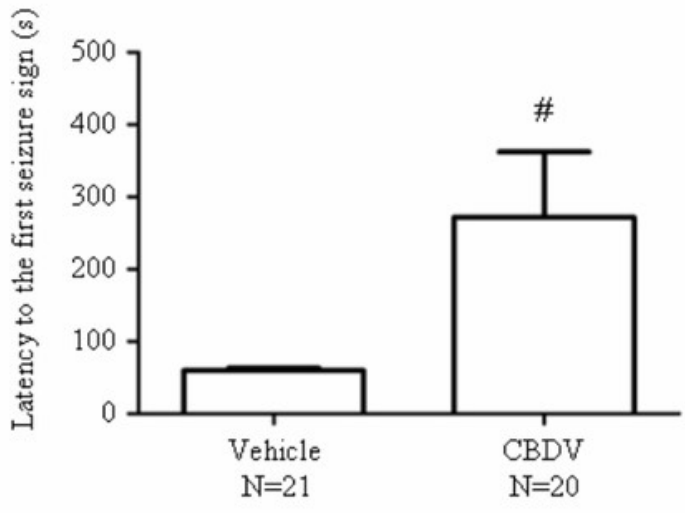

D

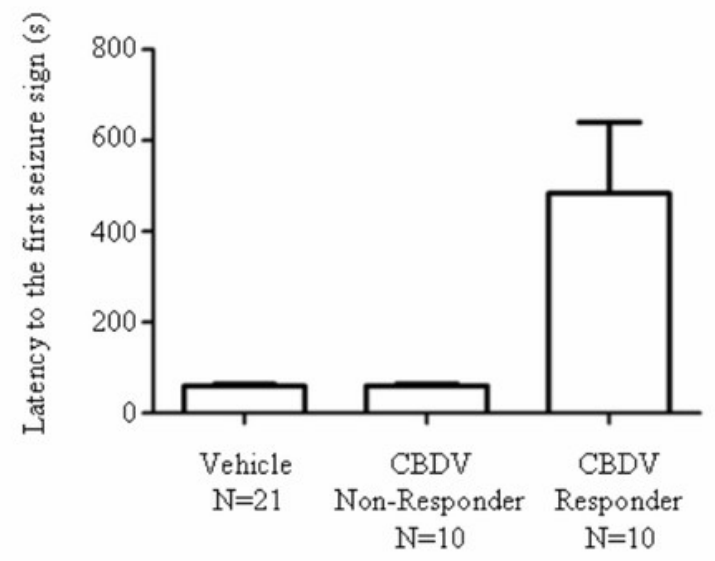




\section{Figure 2}

Correlation analysis between seizure severity and mRNA expression levels in the hippocampus.

Correlations between mRNA expression of Fos (A), Egr1 (B), Arc (C), Bdnf (D) and Ccl4 (E) and seizure severity were analysed using Spearman's rank correlation coefficient.
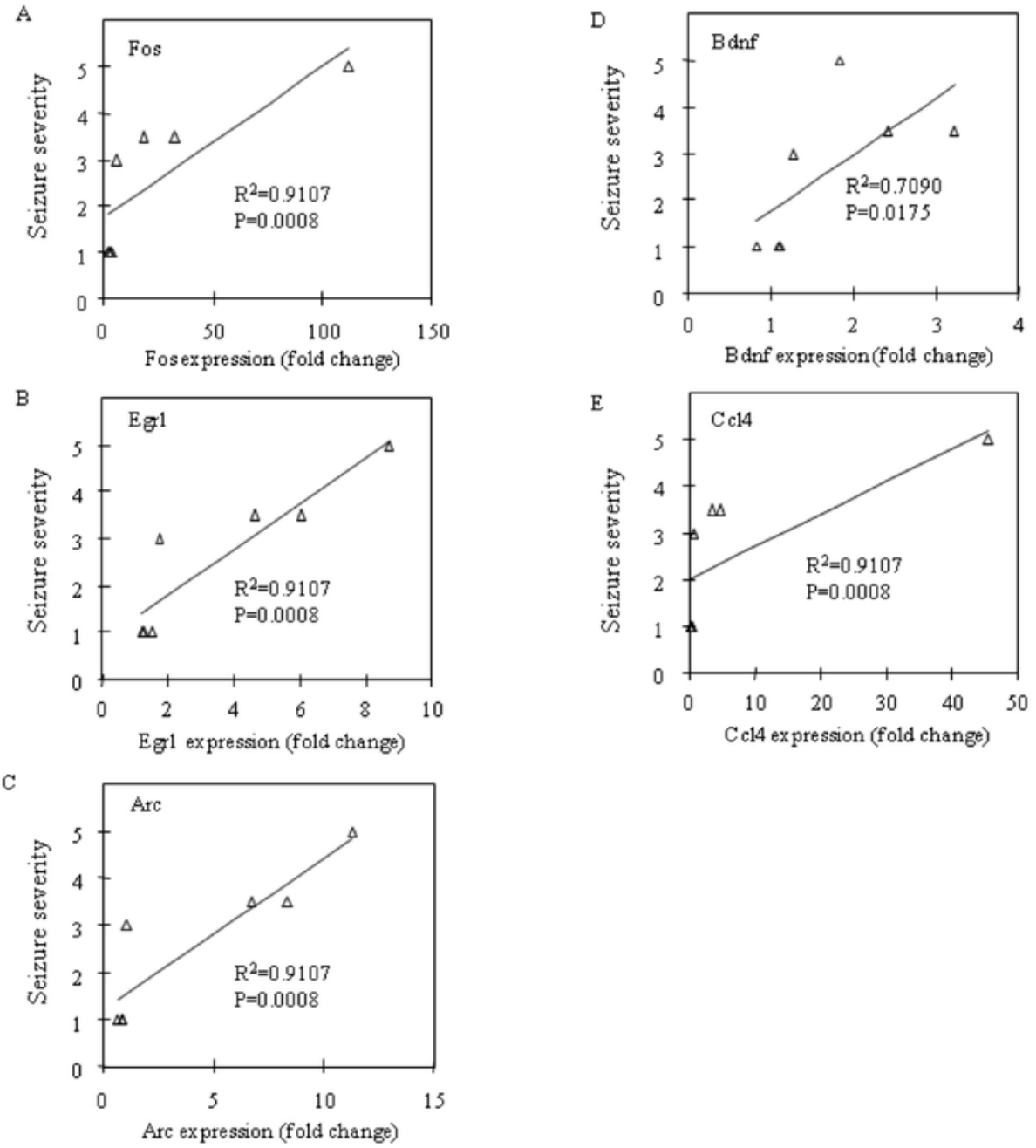


\section{Figure 3}

Correlation analysis between seizure severity and mRNA expression levels in the neocortex.

Correlations between mRNA expression of Fos (A), Egr1 (B), Arc (C), Bdnf (D) and Ccl4 (E) and seizure severity were analysed using Spearman's rank correlation coefficient.
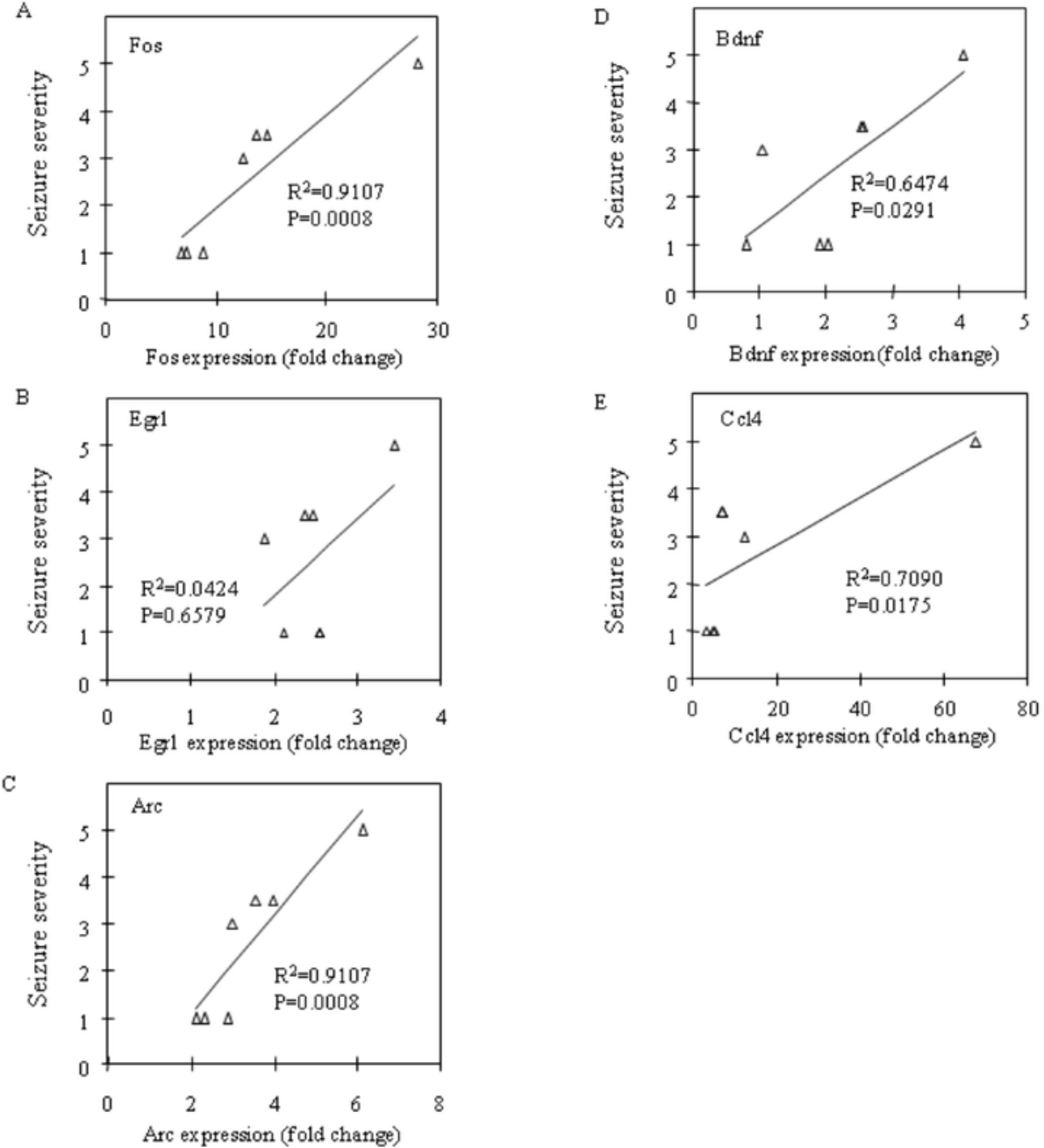


\section{Figure 4}

Correlation analysis between seizure severity and mRNA expression levels in the prefrontal cortex.

Correlations between mRNA expression of Fos (A), Egr1 (B), Arc (C), Bdnf (D) and Ccl4 (E) and seizure severity were analysed using Spearman's rank correlation coefficient.
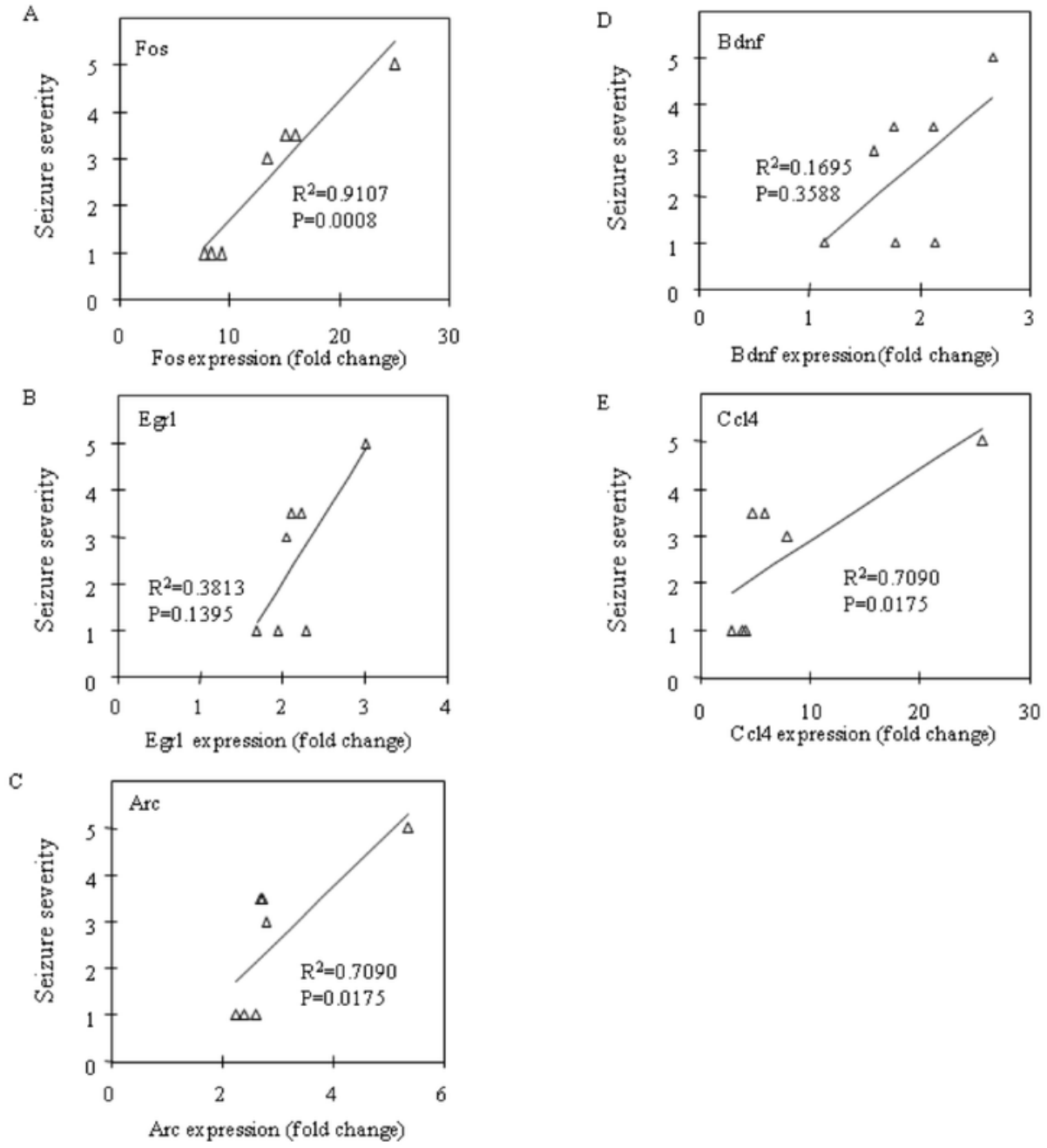


\section{Figure 5}

Subgroup-analysis of mRNA levels of epilepsy-related genes in CBDV responders and nonresponders.

Subgrouping CBDV+PTZ treated animals into responders (criterion: seizure severity $\leq 3.25$ ) and non-responders (criterion: seizure severity $>3.25$ ) revealed that the PTZ-induced increases of mRNA expression of Fos (A), Egr1 (B), Arc (C), Bdnf (D) and Ccl4 (E) were significantly suppressed in brain regions examined from the CBDV responder subgroup . mRNA levels are presented as a fold change vs mean level of vehicle+saline treated group (data are expressed as mean \pm s.e.m.). *: $\mathrm{P}<0.05$ by t-test vs vehicle+PTZ group.
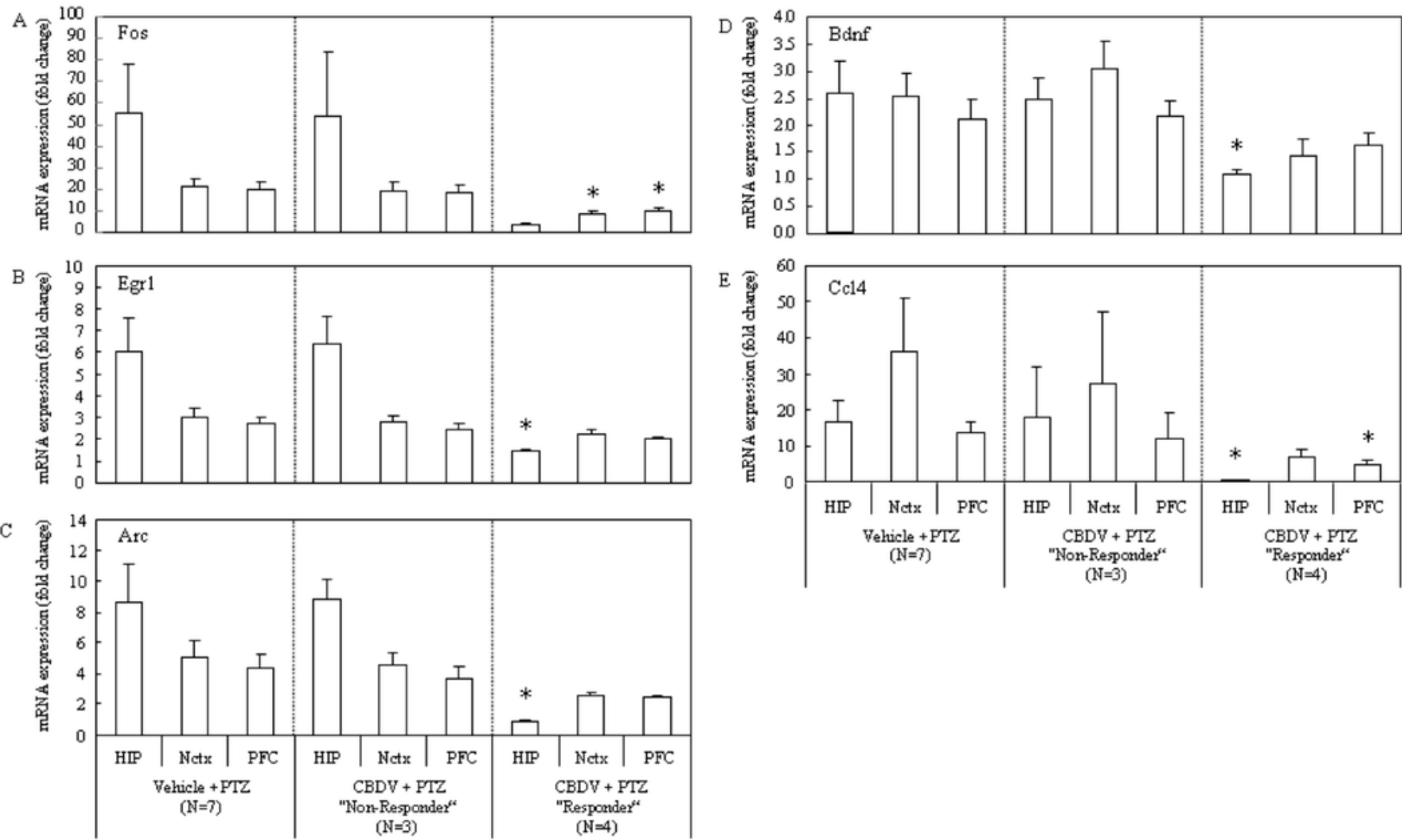